\title{
Resistance profiles of urinary tract infections in general practice - an observational study
}

Guido Schmiemann 1* Ildikó Gágyor², Eva Hummers-Pradier² and Jutta Bleidorn ${ }^{3}$

\begin{abstract}
Background: Guideline recommendations on therapy in urinary tract infections are based on antibiotic resistance rates. Due to a lack of surveillance data, little is known about resistance rates in uncomplicated urinary tract infection (UTI) in general practice in Germany. In a prospective observational study, urine cultures of all women presenting with urinary tract infections in general practice were analysed. Resistance rates against antibiotics recommended in German guidelines on UTI are presented.

Methods: In a prospective, multi-center observational study general practitioner included all female patients $\geq$ 18 years with clinically suspected urinary tract infection. Only patients receiving an antibiotic therapy within the last two weeks were excluded.
\end{abstract}

Results: 40 practices recruited 191 female patients (mean age 52 years; range 18-96) with urinary tract infections. Main causative agent was Escherichia coli (79\%) followed by Enterococcus faecalis (14\%) and Klebsiella pneumoniae (7.3\%).

Susceptibiliy of E.coli as the main causative agent was highest against fosfomycin and nitrofurantoin, with low resistance rates of 4,5\%; 2,2\%. In 17,5\%, E.coli was resistant to trimethoprim and in 8,5\% to ciprofloxacin.

Conclusions: Resistance rates of uropathogens from unselected patients in general practice differ from routinely collected laboratory data. These results can have an impact on antibiotic prescribing and treatment recommendations.

Keywords: Urinary tract infection, Primary care, Drug resistance, Anti-bacterial agents

\section{Background}

Antibiotic resistance is an emerging and serious public health problem resulting in increased morbidity and mortality. In urinary tract infections (UTI), resistance rates against commonly prescribed antimicrobial agents are constantly rising. Nowadays, in many countries more than $20 \%$ of responsible uropathogens are resistant to trimethoprim /sulfamethoxazole (tmp-smx) and to cephalosporins. This increasing resistance is also being observed for fluoroquinolones with resistance rates, risen up to $10 \%[1,2]$. As medicine faces a bleak outlook on availability of effective antibiotic treatment, new therapeutic strategies for urinary tract infections are necessary.

\footnotetext{
* Correspondence: schmiemann@uni-bremen.de

${ }^{1}$ Department for Health Services Research, Institute for Public Health and

Nursing Science, Universität Bremen, Bremen, Germany

Full list of author information is available at the end of the article
}

In 2011 an action plan has been launched by the European Commission [3] to tackle these problems. Aims and strategies include the promotion of a restrictive and appropriate use of antibiotics as well as the promotion of national surveillance programs. To optimize antibiotic treatment of uncomplicated UTI, the latter is urgently required for several reasons:

- Although the majority of antibiotic prescribing for uncomplicated UTI takes place in primary care, information on antibiotic resistance is mainly based on data from hospitals or laboratories, i.e. - highly selected patients. Thus, results cannot be generalized to UTI patients in general practice who are likely to present with lower resistance rates.

- In general practice, urine sample of patients with UTI are not routinely tested for resistance patterns. According to German guidelines on UTI [4,5],

\section{() Biomed Central}


resistance testing is recommended only in cases of treatment failure or suspected complications.

- Resistance patterns of causative uropathogens are known to vary considerably between regions and countries [1].

In Germany, as in many other countries, there is no sentinel network which routinely assesses resistance rates of urinary tract infections in general practice [6].

In face of all these facts, a prospective observational study to target antibiotic therapy in uncomplicated UTI tackles the following questions:

Which uropathogens cause uncomplicated UTI in general practice?

To what extent are these uropathogens susceptible to antibiotics recommended in German guidelines?

\section{Method}

The study idea emerged in a web based discussion forum of German general practitioners hosted by the German College of General Practitioners and Family Physicians. A prospective multi-center observational study design was set up. 579 physicians participating in the web based discussion forum and 193 teaching practices of the Institute of General Practice of Hannover Medical School were invited for participation via e-mail.

During a six week period in autumn 2011, participating practices were asked to include all female patients $\geq$ 18 years in whom urinary tract infection was suspected. The only exclusion criterion was antibiotic therapy within the last two weeks.

After written consent was obtained, patients were asked to provide a clean catch midstream urine sample. The sample was sent to the local laboratory. All laboratories were asked to perform resistance testing at least for trimethoprim, ciprofloxacin, nitrofurantoin and fosfomycin, even if these antibiotics were not being tested routinely.

For classification of UTI and prevailing risk factors, patient data on age, pregnancy status, indwelling urine catheter, risk factors for a complicated infection (i.e. hospital stay within the last 2 weeks, immunosuppression, or neurological disease affecting micturition), and previous UTI were documented by the general practitioners (GP) from patients' records.

Patient data and laboratory results were entered into a web based survey instrument (lime-survey ${ }^{\circledR}$ ) in anonymous form. Access to the database was restricted by a personal access token and performed by medical staff. The patients themselves had no access to the database.

For financial reasons - the study was conducted without external funding - a central laboratory was not feasible. Laboratories conducting urine analyses have to be certified and have to use standardized methods, either
DIN, EuCast or CLSI. All urine cultures/susceptibility tests were performed in the laboratories the practices were attached to. Susceptibility of the causative bacteria was categorized as susceptible (S), intermediate (I) or resistant ( $\mathrm{R})$.

Quality control: a randomized sample of $5 \%$ of all patients $(\mathrm{n}=14)$ was drawn by the investigators. A study assistant compared patient data and results of susceptibility testing entered in the web based survey with the original data held on file in the practice. The comparison was based on a re-identification code known to the practice only. Neither causative agents nor susceptibility results had to be corrected due to the quality control.

Descriptive data analysis (absolute and relative frequencies) was performed descriptively using Microsoft Excel $^{(}$.

Microbiological methods: Native midstream urine was sent to the laboratories. Laboratories were asked to perform a routine urine culture and identification of pathogens as well as susceptibility testing including trimethoprim, fosfomycine-trometamol, nitrofurantoin and ciprofloxacin. These agents were chosen as they are recommended by national guidelines [5]. Positive urine culture was defined as bacterial count $\geq 10^{3} \mathrm{cfu} / \mathrm{ml}$.

Approval of the ethics committee of Hannover Medical School was obtained (N0. 1138 2011).

\section{Results}

\section{Participating practices/patient recruitment}

67 practices expressed their willingness to participate, 40 practices included patients ( $\varnothing 4.8$ patients/practice). Patient recruitment was not possible for some practices $(\mathrm{n}=4)$ as their laboratory would not provide the recommended antibiotic tests against trimethoprim or fosfomycin. [Most of the participating practices were from Lower Saxony (28/40).]

Within the six week study period, most practices $(\mathrm{n}=25)$ included $1-5$ patients; 13 practices included 6-10 patients and two practices included more than 11 patients.

\section{Patient characteristics}

191 women with positive urine culture were included. The mean age was 51.6 years (SD 21.7; Range 18-96). The most prevalent risk factors for UTI were diabetes mellitus (9.6\%; 18/191) and recurrent UTI 18.3\% $(\mathrm{n}=35)$. (see Table 1 for details).

\section{Urine culture and uropathogens}

In $36.1 \%$ of urine cultures, more than one species could be identified; relevant uropathogens from all cultures were included.

Escherichia coli was found to be the causative pathogen in $72.8 \%$ (139/191). Other typical uropathogens 
Table 1 Patient characteristics

\begin{tabular}{ll}
\hline Age & \% Patients $(\mathbf{n}=\mathbf{1 9 1 )}$ \\
\hline Diabetes mellitus & $51.6(S D 21.7$, Range 18-96) \\
\hline Recurrent UTI & $9.6 \%$ \\
\hline Pregnant & $18.3 \%$ \\
\hline Indwelling urinary catheter & $0 \%$ \\
\hline Other risk factor & $0 \%$ \\
\hline
\end{tabular}

Characteristics of 191 female patients with urinary tract infections.

$\mathrm{SD}=$ standard deviation, Recurrent UTI = Previous Urinary tract infection in the last 6 month; Other risk factors = hospital stay within the last 2 weeks, immunosuppression or neurological disease affecting micturition.

found were Enterococcus faecalis ( $\mathrm{n}=26 ; 13.6 \%)$, Klebsiella pneumoniae $(n=14 ; 7.3 \%)$, Proteus mirabilis $(n=11)$ (see Table 2).

\section{Susceptibility testing}

Susceptibiliy of E.coli as the main causative agent was highest against fosfomycin and nitrofurantoin, with low resistance rates of $4,5 \%$ resp. $2,2 \%$. In $17,5 \%$, E.coli was resistant to TMP and in $8,5 \%$ to ciprofloxacin. In contrast to E.coli, other typical uropathogens showed higher resistance rates for TMP, fosfomycin and nitrofurantoin (50\%, 21.7\%, 23.8\%).

Ciprofloxacin is equally effective against E. coli and other typical uropathogens (8.7\%; 8.1\%). Further results on susceptibility are presented in detail in Table 2.

In a few cases a urine culture was not tested for all antibiotics requested. This refers mainly to trimethoprim (130/139 samples were tested) and reflects the lack of a standard in antibiotic testing among laboratories (see Table 2).

\section{Recurrent UTI}

35 patients had experienced a previous urinary tract infection in the last six months. Besides E.coli (29/35), causative pathogens in recurrent infections were Staphylococcus aureus, Enterococcus faecalis, Proteus mirabilis, Staphylococcus saprophyticus and Klebsiella pneumoniae.

In recurrent UTI, E.coli showed higher resistance rates against trimethoprim (25\%) and ciprofloxacin (17\%) while resistance rates for nitrofurantoin (3.4\%) and fosfomycin $(0 \%)$ were very low.

\section{Discussion}

In this prospective observational study, causative agents and susceptibility results in uncomplicated UTI in general practice were collected with a pragmatic approach. Though participating practices constitute a (selfselected) convenience sample which cannot be considered representative, practices were located widely across (predominately north western) Germany. Pragmatic inclusion criteria were given for patients to facilitate recruitment, based on the GPs clinical judgment, which is usually based on patient history and symptoms. While participating practices are likely to be more interested in research (and possibly in antibiotic resistance) than nonparticipating or non-recruiting practices, it seems unlikely that their patients are different [7].

Thus, general practice based information on resistance patterns of typical uropathogens in general practice in Germany can be provided for the first time. Comparing our results with reports of routinely collected data from a more specialized level of care [8,9] revealed relevant differences (Table 3). Antibiotic resistance against trimethoprim, a drug recommended by many guidelines

Table 2 Bacteria detected and susceptibility profile

\begin{tabular}{|c|c|c|c|c|c|c|c|c|c|c|c|c|c|}
\hline \multirow[t]{2}{*}{ Isolates } & \multirow[t]{2}{*}{ n (\%) } & \multicolumn{3}{|c|}{ Trimethoprim } & \multicolumn{3}{|c|}{ Fosfomycin } & \multicolumn{3}{|c|}{ Nitrofurantoin } & \multicolumn{3}{|c|}{ Ciprofloxacin } \\
\hline & & S n (\%) & I n (\%) & $\mathrm{R}$ n (\%) & $\mathrm{S}$ n (\%) & I n (\%) & R n (\%) & $\mathrm{S}$ n (\%) & I n (\%) & R n (\%) & $\mathrm{S}$ n (\%) & I n (\%) & R n (\%) \\
\hline All & $191(100)$ & & & & & & & & & & & & \\
\hline E. coli & $139(72.7)$ & $105(80.8)$ & $2(1.7)$ & $23(17.5)$ & $126(95.5)$ & $0(0)$ & $6(4.5)$ & $130(94.2)$ & $5(3.6)$ & $3(2.2)$ & $126(91.3)$ & $0(0)$ & $12(8.7)$ \\
\hline $\begin{array}{l}\text { Other typical } \\
\text { uropathogens }\end{array}$ & $71(37.2)$ & $28(50)$ & 0 & $28(50)$ & $46(76,8)$ & $1(1.7)$ & $13(21.7)$ & $43(68.2)$ & $5(7.9)$ & $15(23.8)$ & $48(77.4)$ & $9(14.5)$ & $5(8.1)$ \\
\hline $\begin{array}{l}\text { Enterococcus } \\
\text { faecalis }\end{array}$ & 26 (13.6) & 6 & 0 & 14 & 15 & 1 & 6 & 21 & 0 & 2 & 16 & 5 & 2 \\
\hline Proteus mirabilis & $11(5.7)$ & 1 & 0 & 8 & 8 & 0 & 0 & 0 & 0 & 10 & 9 & 0 & 0 \\
\hline $\begin{array}{l}\text { Staph. } \\
\text { saprophyticus }\end{array}$ & $4(2.1)$ & 4 & 0 & 0 & 3 & 0 & 1 & 4 & 0 & 0 & 4 & 0 & 0 \\
\hline Staph. others & $5(2.6)$ & 1 & 0 & 2 & 3 & 0 & 0 & 1 & 2 & 0 & 2 & 1 & 0 \\
\hline $\begin{array}{l}\text { Strept. } \\
\text { agalacticae }\end{array}$ & $8(4.2)$ & 2 & 0 & 3 & 7 & 0 & 0 & 7 & 0 & 0 & 2 & 3 & 2 \\
\hline $\begin{array}{l}\text { Kleb } \\
\text { pneumoniae }\end{array}$ & $14(7.3)$ & 12 & 0 & 1 & 10 & 0 & 4 & 9 & 2 & 3 & 13 & 0 & 1 \\
\hline Staph. aureus & $3(1.6)$ & 2 & 0 & 0 & 0 & 0 & 2 & 1 & 1 & 0 & 2 & 0 & 0 \\
\hline
\end{tabular}

In the first column typical uropathogens and the number of isolates are documented. In the upper two rows results of susceptibility testing against E. coli and all other typical uropathogens are presented. Due to the low numbers no percent rates are given in the remaining rows. 
Table 3 Resistance rates in different settings

\begin{tabular}{lllll}
\hline & $\begin{array}{l}\text { Our } \\
\text { results }\end{array}$ & $\begin{array}{l}\text { Robert Koch } \\
\text { Institute [7] }\end{array}$ & $\begin{array}{l}\text { Antibiotic resistance } \\
\text { monitoring in } \\
\text { Lower Saxony [8] }\end{array}$ & $\begin{array}{l}\text { ARESC } \\
\text { [13] }\end{array}$ \\
\hline TMP & 17.5 & 29.2 & 28.7 & 25.9 \\
\hline Nitrofurantoin & 2.2 & n.a & 1.2 & 4.5 \\
\hline Fosfomycin & 4.5 & n.a & n.a & 0.8 \\
\hline Ciprofloxacin & 8.7 & 18.2 & 13.9 & 4.5 \\
\hline
\end{tabular}

With exception of our study all others used TMP-SMX. N.A = No information on susceptibility rates given.

on urinary tract infections $[5,10,11]$ is obviously lower in general practice patients compared with laboratory surveillance data. Ciprofloxacin, an antibiotic recommended for complicated infections, also shows higher susceptibility in primary care. Fosfomycin and nitrofurantoin still have remarkably low resistance rates, probably due to quite low prescription rates in Germany. Our results confirm that urine cultures submitted to laboratories do not represent the susceptibility patterns in unselected patients $[12,13]$ since in these patients urine cultures are only requested if the patient fails to respond to treatment, has recurrent episodes or other complicating factors. Thus, in the primary care setting, urine cultures for suspected UTI remain exceptional and represent complicated rather than typical uncomplicated patients. Therefore, data from laboratory results $[8,9]$ or studies including data from patients in specialist care (urology, gynecology) [14] differ from our results, they do not represent the antimicrobial resistance situation in general practice in Germany.

The strength of our study is its pragmatic approach in the general practice setting, resulting in a high number of (uncomplicated) patients recruited in a very short period of time. A direct comparison with two epidemiological German studies $[14,15]$ is limited as these studies included only women up to 65 years under specialists care. This may well explain the low number of patients with diabetes mellitus (4.8\%) found by Wagenlehner compared with $9.6 \%$ in our study including elderly women. The lack of a central laboratory resulting in different sets of antibiotics used for routine susceptibility testing, or available for testing at all proved a limit in obtaining comparable susceptibility data for all patients within our study. However, pooling results from different certified laboratories is routinely done when reporting of antimicrobial resistance rates [8]. Therefore the fact of using pooled data from different local laboratories instead of a central one is unlikely to bias our results. In any case, it reflects usual practice and usual community based care in Germany.

National or regional standards for the selection of antibiotics to be included in susceptibility analysis are missing in Germany, as well as legal pressure to include antibiotics recommended in guidelines. In many microbiological laboratories, routinely conducted tests often do not include all antimicrobial agents recommended by current guidelines, for example, fosfomycin-trometamol or nitrofurantoin are often missing. This constitutes a very relevant barrier against guideline implementation and appropriate use of antibiotics.

Resistance rates of common uropathogens have an important impact on guideline recommendations regarding the choice of antibiotics. Although there is no linear correlation between resistance level and strength of a recommendation, rising resistance rates compromise acceptance of a recommendation, and confirmed lower resistance profiles imply the need to review a guideline recommendation. Based on expert consensus, usually a resistance level of $>20 \%$ is used as a cut-off in guidelines on urinary tract infections $[16,17]$.

With a resistance rate of $17.5 \%$ for the main causative agent E. coli, our results confirm the recommendation of TMP as a first choice antibiotic substance in the German primary care setting.

An urgent task for the future is to build up a sentinel network. Only by collecting resistance data permanently, a tailored and specific antibiotic therapy of UTI is permitted. This also implies implementation of guidelineadjusted resistance testing in medical laboratories.

\section{Conclusions}

Resistance rates of uropathogens from unselected patients in general practice differ from routinely collected data from laboratories. These results have a major effect on antibiotic prescribing and treatment recommendations. Sentinel networks for a representative UTI resistance data are urgently needed to monitor UTI resistances in general practice.

\section{Competing interests}

All authors declare that they have no competing interests.

\section{Authors' contributions}

GS and JB conceived the design of the study with contribution from IG and EHP. GS drafted the manuscript and JB, IG and EHP contributed to the manuscript. All authors read and approved the final manuscript.

\section{Achnowledgements}

We gratefully thank all participating practices. Thanks to Daniel Dettmer for his support in data management.

\section{Author details}

${ }^{1}$ Department for Health Services Research, Institute for Public Health and Nursing Science, Universität Bremen, Bremen, Germany. ${ }^{2}$ Department of General Practice and Family Medicine, University of Goettingen, Göttingen, Germany. ${ }^{3}$ Institute of General Practice and Family Medicine, Hanover Medical School, Hannover, Germany.

Received: 31 May 2012 Accepted: 18 November 2012

Published: 21 November 2012 


\section{References}

1. Schito GC, Naber KG, Botto H, Palou J, Mazzei T, Gualco L, et al: The ARESC study: an international survey on the antimicrobial resistance of pathogens involved in uncomplicated urinary tract infections. Int J Antimicrob Agents 2009, 34(5):407-413.

2. De Backer D, Christiaens T, Heytens S, De Sutter A, Stobberingh EE, Verschraegen G: Evolution of bacterial susceptibility pattern of Escherichia coli in uncomplicated urinary tract infections in a country with high antibiotic consumption: a comparison of two surveys with a 10 year interval. J Antimicrob Chemother 2008, 62(2):364-368.

3. Watson R: Europe launches 12 point plan to tackle antimicrobial resistance. BMJ 2011, 343:d7528. 21.

4. Wagenlehner FME, Schmiemann G, Hoyme U, Fünfstück R, HummersPradier E, Kaase M: S3 Guidelines: epidemiology, diagnosis, treatment and management of uncomplicated community-acquired bacterial urinary tract infections in adult patients. Nieren Hochdruckkrankheiten 2011, 40(1):2-20.

5. Schmiemann G, Gebhardt K, Matejczyk M, Hummers-Pradier E (Eds): Brennen beim wasserlassen - Anwenderversion der S3 Leitlinie Harnwegsinfekte. Düsseldorf: Omicron publishing; 2009.

6. Hoffmann K, Wagner G, Apfalter P, Maier M: Antibiotic resistance in primary care in Austria - a systematic review of scientific and grey literature. BMC Infect Dis 2011, 11:330. 28.

7. Muller S, Wynne-Jones G, Daniel R, Creavin ST, Bishop A, Mallen CD: There is no association between a measure of clinical care and the response rate of GPs to postal surveys: a methodological study. Eur J Gen Pract 2012, 18(3):154-158.

8. Robert Koch Institut: Antibiotika Resistenz Surveillance. https://ars.rki.de.

9. Antibiotika-Resistenz-Monitoring in Niedersachsen (ARMIN). http://www.nlga. niedersachsen.de/portal/live.php?navigation_id=6599\&article_id=19418\&_ psmand $=20$.

10. van Pinxteren B, van Vliet SM, Wiersma TJ, Goudswaard AN, Nederlands Huisartsen Genootschap: Summary of the practice guideline 'Urinary-tract infections' (second revision) from the Dutch college of general practitioners. Ned Tijdschr Geneeskd 2006, 150(13):718-722. 1.

11. Wuorela M, Kouri T, Laato M, Lipponen P, Sammalkorpi K, Uhari M, Uusitalo $L$, Vuento R: Update on current care guidelines: urinary tract infections. Duodecim 2011, 127(21):2334-2335.

12. Tan TY, McNulty C, Charlett A, Nessa N, Kelly C, Beswick T: Laboratory antibiotic susceptibility reporting and antibiotic prescribing in general practice. J Antimicrob Chemother 2003, 51(2):379-384

13. Ti TY, Kumarasinghe G, Taylor MB, Tan SL, Ee A, Chua C, et al: What is true community-acquired urinary tract infection? Comparison of pathogens identified in urine from routine outpatient specimens and from community clinics in a prospective study. Eur $J$ Clin Microbiol Infect Dis 2003, 22(4):242-245.

14. Wagenlehner FM, Wagenlehner C, Savov O, Gualco L, Schito G, Naber KG: Clinical aspects and epidemiology of uncomplicated cystitis in women. German results of the ARESC Study. Urologe A 2010, 49(2):253-261.

15. Kahlmeter G, Poulsen HO: Antimicrobial susceptibility of Escherichia coli from community-acquired urinary tract infections in Europe: the ECO. SENS study revisited. Int J Antimicrob Agents 2012, 39(1):45-51.

16. Naber KG, Bergman B, Bishop MC, Bjerklund-Johansen TE, Botto H, Lobel B, et al: EAU guidelines for the management of urinary and male genital tract infections. Urinary Tract Infection (UTI) Working Group of the Health Care Office (HCO) of the European Association of Urology (EAU). Eur Uro 2001, 40(5):576-588.

17. Wagenlehner FME, Schmiemann G, Hoyme U, Fünfstück R, HummersPradier E, Kaase M, et al: National S3 guideline on uncomplicated urinary tract infection: recommendations for treatment and management of uncomplicated community-acquired bacterial urinary tract infections in adult patients. Urologe Ausgabe A 2011, 50(2):153-169.

doi:10.1186/1471-2490-12-33

Cite this article as: Schmiemann et al:: Resistance profiles of urinary tract infections in general practice - an observational study. BMC Urology 2012 12:33.

\section{Submit your next manuscript to BioMed Central and take full advantage of:}

- Convenient online submission

- Thorough peer review

- No space constraints or color figure charges

- Immediate publication on acceptance

- Inclusion in PubMed, CAS, Scopus and Google Scholar

- Research which is freely available for redistribution 